\title{
PRIMEROS DATOS SOBRE LOS HÁBITOS ALIMENTARIOS DEL TIGRILLO, Leopardus pardalis, EN UN BOSOUE ANDINO DE COLOMBIA
}

\author{
FIRST DATA ON THE FOOD HABITS \\ OF THE OCELOT, LeOpardus pardalis, \\ IN A COLOMBIAN ANDEAN FOREST
}

\author{
Francisco Sánchez ${ }^{1}$ \\ Bibiana Gómez-Valencia ${ }^{2}$ \\ Silvia J. Álvarez ${ }^{3}$ \\ Marcela Gómez-Laverde ${ }^{4}$
}

\section{RESUMEN}

El estudio de siete muestras fecales de tigrillo, Leopardus pardalis, encontradas en un bosque andino colombiano, entre 2200 y $2900 \mathrm{~m}$ de altitud, permitió obtener una visión preliminar de los hábitos alimentarios de este felino. Leopardus pardalis consumió principalmente mamíferos, $\sim 90 \%$ de las presas y también aves. Entre los mamíferos, los de $<400 \mathrm{~g}$ y de $>400 \mathrm{~g}$ representaron $\sim 52 \%$ y $\sim 38 \%$ de las presas, respectivamente. La frecuencia relativa de presas de $>400 \mathrm{~g}$ es alta comparada con lo presentado por otros estudios;

${ }^{1}$ Ph.D. Instituto de Ciencias Naturales-Departamento de Biología, Universidad Nacional de Colombia. Ciudad Universitaria. Dirección actual y para correspondencia: Facultad de Ciencias Ambientales, Universidad de Ciencias Aplicadas y Ambientales, U.D.C.A. Calle 222 \# 55-37 Bogotá, Colombia. Correo electrónico: fasbos@gmail.com.

${ }^{2}$ Bióloga. Instituto de Ciencias Naturales-Departamento de Biología, Universidad Nacional de Colombia. Ciudad Universitaria, Bogotá, Colombia. Correo electrónico: bibianagomez_valencia@yahoo.com.

3 M.Sc. Fundación Caipora. Calle 129b \# 51 - 91. Bogotá. Correo electrónico: silviaalvarez@fundacioncaipora.org.

${ }^{4}$ M.Sc. Instituto de Ciencias Naturales-Departamento de Biología, Universidad Nacional de Colombia. Ciudad Universitaria, Bogotá, Colombia. Correo electrónico: gomezlaverde@supercabletv.net.co. posiblemente, se deba a que depredadores de mayor tamaño han desaparecido del área de estudio, lo que permite al tigrillo ampliar su rango de tamaño de presas, al convertirse en el mayor depredador del bosque.

Palabras clave: Andes, departamento Caldas, carnívora, dieta, Felidae.

\section{SUMMARY}

A preliminary analysis of the feeding habits of the ocelot, Leopardus pardalis, was made based on the examination of seven fecal samples found in a Colombian Andean forest, between 2200 and $2900 \mathrm{~m}$ of altitude. The ocelot fed mainly on mammals, $\sim 90 \%$ of prey, and also ate birds. Among mammals, those weighing $<400 \mathrm{~g}$ and $>400 \mathrm{~g}$ represented $\sim 52 \%$ and $38 \%$ of the prey, respectively. The relative frequency of $>400 \mathrm{~g}$ prey is high when compared with the results from other studies. This might be due to the extinction of larger predators from the study site, which consequently allowed the ocelot to widen its range of prey sizes when it became the largest predator of the forest.

Key words: Andes, Caldas, Carnivora, diet, Felidae.

\section{INTRODUCCIÓN}

El tigrillo, Leopardus pardalis, Linneo 1758 (Carnivora: Felidae), se distribuye en el Nuevo Mundo desde 
Arizona y Texas, en Norte América, a través de Centro America, hasta Argentina y Uruguay, en América del Sur (Wozencraft, 1993). Este felino habita diferentes ecosistemas, entre los que se incluyen sabanas, bosques secos y lluviosos de tierras bajas, así como bosques secos y lluviosos premontanos y montanos (Bisbal, 1989). El tigrillo es más frecuente en tierras bajas que en áreas montanas (Bisbal, 1989; Eisenberg, 1989) e incluso algunos autores mencionan que en Colombia no se halla por encima de los $2400 \mathrm{~m}$ de altura (Alberico et al. 2000). Por esto, aunque el tigrillo es la especie mejor estudiada entre los felinos pequeños de América, es poca la información acerca de su biología en regiones montañosas y, en particular, no hay datos sobre su dieta en hábitat andinos (Murray \& Gardner, 1997).

Los tigrillos adultos tienen entre 8 y $12 \mathrm{~kg}$ de masa corporal (Emmons, 1997) y cazan presas con un amplio rango de tamaños. Los estudios disponibles sobre la dieta del tigrillo, que se han realizado en bosques tropicales de zonas bajas, indican que se alimenta principalmente de vertebrados pequeños, <400g, entre los que se cuentan: pequeños mamíferos, aves, reptiles, peces y anfibios (Bisbal, 1986; Mondolfi, 1986; Emmons, 1987; Ludlow \& Sunquist, 1987; Konecny, 1989; Chinchilla, 1997; De Villa Mesa et al. 2002; Bianchi E Mendes, 2007; Abreu et al. en prensa). También caza invertebrados y animales de mayor tamaño, como roedores caviomorfos $(>3 \mathrm{~kg})$, por ejemplo, Dasyprocta y Agouti; primates ( $>3 \mathrm{~kg}$ ), como Alouatta y Brachiteles y artiodáctilos ( $>5 \mathrm{~kg}$ ), tales como saínos y venados. Así, cabe preguntar si los hábitos alimentarios de $L$. pardalis en regiones andinas difieren de los exhibidos en zonas bajas neotropicales. Durante un estudio sobre los mamíferos en un bosque en los Andes Centrales de Colombia (Sánchez E Alvear, 2003; Sánchez et al. 2004), se recolectaron siete muestras de heces de tigrillo, entre 2200 y 2900 m de altitud. Aunque el número de muestras es pequeño, debido a la baja abundancia de estos felinos en el área de estudio (Sánchez et al. 2008), estas muestras se examinaron para obtener los primeros datos sobre la dieta del tigrillo en bosques andinos. Este tipo de información es particularmente importante, dado que el tigrillo es una especie casi amenazada en Colombia (Jorgenson et al. 2006), y una de las razones, para ello, es la destrucción de los hábitat boscosos, muy acentuada en los Andes (Kattan E Álvarez-López, 1996).

\section{MATERIALES Y METODOS}

Área de estudio: La investigación, se realizó en la Reserva Río Blanco, municipio de Manizales, departmento de Caldas, Colombia. La Reserva tiene un área aproximada de $30 \mathrm{~km}^{2}$, entre 2150 y $3750 \mathrm{~m}$ de altitud y comprende un remanente de bosque rodeado de pastizales y áreas cultivadas, conectada con otras zonas de bosque a menores alturas, por remanentes de bosques riparios. El principal tipo de vegetación es el bosque andino, pero también hay reforestaciones con aliso (Alnus acuminata) y con especies exóticas (Eucaliptus sp., Pinus sp., Cupresus sp.). Como en otras zonas de la Cordillera Central, el aliso ha sido usado para recuperar la cobertura boscosa y para proteger fuentes de agua (Cavelier, 1995; Murcia, 1997) y comprende aproximadamente $26 \%$ del área de la Reserva, mientras que las reforestaciones, con especies exóticas, comprenden aproximadamente el 21\%. En la Reserva también hay áreas en regeneración dominadas por pastos, las cuales presentan árboles dispersos.

Las áreas de la Reserva con bosque andino tienen vegetación con dos estratos de árboles (5-15m y 20$25 \mathrm{~m}$ ) y los árboles y arbustos del sotobosque tienen hasta 5m (Anónimo, 1987). Los árboles tienen copas en forma de sombrilla, con los troncos y ramas cubiertas por epífitas vasculares y no vasculares (HernándezCamacho E Sánchez, 1992). En general, los árboles tienden a decrecer en tamaño al aumentar la altitud. En estos bosques son abundantes las familias Asteraceae, Melastomataceae, Solanaceae y Ericaceae y, en particular, son abundantes las especies Weinmannia pubescens, Freziera canescens y Saurauia brachybotris (Alvear, 2000). En las reforestaciones, el tamaño de los árboles aumenta con la edad de plantación y forma un dosel uniforme, entre los 20 y $35 \mathrm{~m}$. El sotobosque de las reforestaciones está dominado por especies heliófilas, como Bocconia frutescens. Más detalles sobre la vegetación de la Reserva, se pueden encontrar en Sánchez et al. (2004; 2008).

Muestreo y análisis de las heces: Durante recorridos hechos por caminos existentes en la Reserva, se recogieron las muestras con heces de tigrillo. Los recorridos, se llevaron a cabo, principalmente, entre las 05:00 y 07:00 horas, para un total de 36 recorridos en bosque andino (2500-3300m), 43 en reforestaciónes 
con aliso (2800-3200m) y doce en áreas de regeneración de bosque altoandino (3300-3500m). En las áreas en regeneración no se encontraron heces de tigrillo. Entre los meses de octubre y noviembre de 1998, se encontraron tres muestras, entre enero y abril de 1999, otras tres, y en abril de 2000, se halló la última muestra, que correspondía a una acumulación de deposiciones de un período de tiempo desconocido. Seis de las muestras, se encontraron en tres sitios de la Reserva que formaban pequeñas cavidades y estaban ligeramente elevados $(\sim 20-50 \mathrm{~cm})$ del nivel del camino, es decir, en letrinas.

Para identificar las heces, se usaron las figuras y medidas presentadas en Aranda (1981). La presencia de huellas de tigrillo en las letrinas, reconocidas con base en las figuras y medidas de la misma referencia, sirvió para confirmar la identicación de las heces.

Después de recolectadas en bolsas de papel marcadas, las heces fueron llevadas al laboratorio para ser secadas $\mathrm{y}$, posteriormente, se preservaron en etanol $70 \%$. Para su análisis, las muestras fueron segregadas y examinadas con un microscopio estereoscópico. De cada muestra, se separaron fragmentos de huesos, dientes, garras y pelos. Estas partes, se identificaron por comparación con especímenes capturados en la Reserva (Sánchez E Alvear, 2003; Gómez-Valencia, 2004) y preservados en la Colección de Mamíferos del Instituto de Ciencias Naturales-Universidad Nacional de Colombia, así como con otros ejemplares, incluidos en esta colección. Adicionalmente, se consultó literatura especializada para la identificación de los restos de los pequeños mamíferos (Voss, 1988; Carleton \& Musser, 1989; Voss, 2003). Los nombres comunes de la mayoría de las especies, se obtuvieron de entrevistas con habitantes de la región (Sánchez E Alvear, 2003), pero los nombres de los roedores pequeños, siguen a Rodríguez-Mahecha et al. (1995).

De acuerdo con la presencia de fragmentos de huesos y dientes de cada tipo de presa en cada muestra, se calculó el número mínimo de individuos, NMI (Emmons, 1987). Para ello, se asumió que todos los fragmentos de una misma especie en una muestra pertenecían al mismo individuo, a menos que se encontrara el mismo diente o hueso, más de una vez. El NMI fue usado para estimar la frecuencia relativa de cada tipo de presa, así: $\mathrm{FR}_{i}=[\mathrm{NMI}$ de la presa i / total NMI $] \times 100$. Cuando se encontraron fragmentos de la misma especie de presa en muestras recolectadas en días consecutivos y en la misma letrina, se consideró que dichos fragmentos pertenecían al mismo individuo. No se calculó la frecuencia de ocurrencia de las presas, debido a que una muestra era una acumulación de deposiciones.

La lista de especies, se empleo para clasificar las presas halladas en las muestras, como pequeñas, $<400 \mathrm{~g}$, o medianas $>400 \mathrm{~g}$ y la información sobre la masa de cada especie, se extrajo de referencias bibliográficas (Eisenberg, 1989; Emmons, 1997).

\section{RESULTADOS Y DISCUSIÓN}

Todas las muestras examinadas contenían dos o más tipos de presas y en total se encontró un número mínimo de 29 presas, entre mamíferos y aves (Tabla 1). Los mamíferos pequeños, $<400 \mathrm{~g}$, representaron el $51,72 \%$ de las presas en la dieta del tigrillo, mientras que los mamíferos $>400 \mathrm{~g}$ constituyeron el 37,93\% (Tabla 1). El resto de las presas, $10,34 \%$, se identificaron como aves a partir de huesos y unas pocas plumas, pero no fue posible determinar, con mayor detalle, su identidad taxonómica. De las 29 presas, dos especies representaron casi el $45 \%$ de la dieta: el $27,6 \%$ correspondió a una especie de mamífero pequeño, Microryzomys minutus y un $17,2 \%$, al cusumbo mocoso, Nasuella olivacea.

La principal diferencia en los hábitos alimentarios del tigrillo encontrados en este estudio y los de tierras bajas es la identidad específica de las presas cazadas y esto es porque la mayoría de las presas de este estudio como Didelphis albiventris, Nasuella olivacea, Akodon bogotensis, Chilomys instans, Microryzomys minutus, Thomasomys gr. aureus, Coendou rufescens son propias de ambientes andinos, por encima de los $1500 \mathrm{~m}$ de altitud (Alberico et al. 2000). Adicionalmente, Dinomys branickii también es una presa no antes reportada en la dieta del tigrillo, aunque este roedor está presente tanto en tierras bajas como en áreas montañosas de los Andes, 240-3200m (White E Alberico, 1992).

En la dieta del tigrillo, se encontró un roedor que no había sido registrado en la Reserva (Sánchez E Alvear, 2003; Gómez-Valencia, 2004). En efecto, uno de los dientes hallados en las muestras corresponde a un miembro de la subfamilia Ichtyiominae, pero sus características son 
Tabla 1. Número mínimo de individuos, NMI, en las muestras y frecuencia relativa, FR, de las presas encontradas en las heces del tigrillo en la Reserva Río Blanco, Manizales, Colombia. $\mathrm{FR}_{i}=(\mathrm{NMI}$ del tipo de presa $\mathrm{i} /$ total $\mathrm{NMI}) \times 100$. Muestras $=$ Número de muestras con el tipo de presa $i$.

\begin{tabular}{|c|c|c|c|}
\hline Tipo de presa (Nombre común) & Muestras & NMI & FR \\
\hline Didelphidae $^{1}\left(\right.$ Chucha $\left.^{a}\right)$ & 1 & 1 & 3,45 \\
\hline Akodon bogotensis (Ratoncito arrocero bogotano $0^{b}$ ) & 1 & 1 & 3,45 \\
\hline Chilomys instans (Ratón selvático colombiano $\left.0^{b}\right)$ & 1 & 1 & 3,45 \\
\hline Ichtyiominae & 1 & 2 & 6,90 \\
\hline Microryzomys minutus (Ratoncito arrocero diminuto ${ }^{b}$ ) & 1 & 8 & 27,6 \\
\hline Thomasomys gr. aureus (Ratón montañero acanelado & 1 & 1 & 3,45 \\
\hline Pequeño mamífero no identificado & 1 & 1 & 3,45 \\
\hline Subtotal mamíferos pequeños $(<400 \mathrm{~g})$ & 2 & 15 & 51,72 \\
\hline Didelphis albiventris (Chuchaa) & 1 & 1 & 3,45 \\
\hline Nasuella olivacea (Cusumbo mocosoa) & 3 & 5 & 17,24 \\
\hline Coendou rufescens (Erizo $\left.{ }^{\mathrm{a}}\right)$ & 4 & 3 & 10,34 \\
\hline Dinomys branickii (Guagua lobaa) & 1 & 1 & 3,45 \\
\hline Sylvilagus brasiliensis (Conejo ${ }^{\mathrm{a}}$ ) & 2 & 1 & 3,45 \\
\hline Subtotal mamíferos medianos $(>400 \mathrm{~g})$ & 7 & 11 & 37,93 \\
\hline$\underline{\text { Aves }}$ & 4 & 3 & 10,34 \\
\hline Material vegetal & 4 & & \\
\hline TOTAL & 7 & 29 & $100 \%$ \\
\hline
\end{tabular}

${ }^{1}$ Probablemente es un juvenil de Didelphis albiventris.

${ }^{a}$ Nombre común dado por los habitantes de la región.

${ }^{b}$ Nombre común tomado de Rodríguez-Mahecha et al. (1995).

diferentes a las de Neusticomys monticolus, que es el único representante de la subfamilia, reportado para los Andes Centrales de Colombia (Gómez-Laverde, 1994). Esto sugiere que son necesarios muestreos adicionales para complementar el inventario de mamíferos del área de estudio.

Los resultados indican que el tigrillo se alimentó de especies diurnas, como los cusumbos y las aves, y que también caza animales de hábitos, principalmente nocturnos, como las chuchas, los conejos, los erizos, las guaguas lobas y los pequeños roedores. Estos resultados concuerdan con los estudios que sugieren que este felino está activo básicamente en la noche, pero no es raro que cace en el día (Emmons, 1988; Konecny, 1989; Sunquist et al. 1989).

Como se ha encontrado en estudios anteriores sobre la dieta del tigrillo (Bisbal, 1986; Mondolfi, 1986; Emmons, 1987; Ludlow \& Sunquist, 1987; Konecny, 1989; Chinchilla, 1997; De Villa Mesa et al. 2002; Moreno et al. 2006), este felino consumió presas con un amplio rango de tamaños, desde animales pequeños, como M. minutus, con una longitud cabeza-cuerpo promedio 
de 80,6mm y <18g (Carleton \& Musser, 1989; GómezValencia, 2004), hasta presas como $D$. branickii, cuyos adultos tienen en cabeza-cuerpo entre 730 y 790mm y pesan más de $8 \mathrm{~kg}$ (White $E$ Alberico, 1992). También, como se ha encontrado en estudios anteriores, en la Reserva, el tigrillo se alimentó principalmente de mamíferos, y entre estos los pequeños mamíferos $(<400 \mathrm{~g})$ fueron el tipo de presa mejor representado. Sin embargo, a diferencia de la mayoría de las investigaciones anteriores, la frecuencia de mamíferos medianos, $>400 \mathrm{~g}$, es relativamente alta, $\sim 38 \%$. Una posible explicación a este resultado, se puede relacionar con el pequeño número de muestras en este estudio. Las letrinas donde se encontraron las muestras no estaban alejadas entre sí más de $5 \mathrm{~km}$ y, dado que los tigrillos pueden recorrer más de 6 km por día (Konecny, 1989), es posible que las muestras analizadas pertenecieran al mismo individuo. Así, otra posible explicación para la relativamente alta frecuencia de presas de tamaño mediano es que las muestras analizadas provienen de un animal, con un sesgo por este tipo de presas.

Dado que los tigrillos se comportan como depredadores oportunistas y capturan cualquier vertebrado que puedan cazar (Emmons, 1988; Konecny, 1989), la relativamente alta proporción de mamíferos de $>400 \mathrm{~g}$ en su dieta prodría reflejar la abundancia de estas presas, en el área de estudio. Esta idea es apoyada por el hecho de que el cusumbo mocoso, la presa de $>400 \mathrm{~g}$, más frecuente en las muestras, parece ser uno de los mamíferos medianos más abundantes de la Reserva (Sánchez et al. 2008).

El tigrillo, se ha convertido en el mayor depredador de la Reserva, dado que carnívoros de mayor tamaño, como los pumas, Puma concolor y los jaguares, Panthera onca, desaparecieron de la región, durante el siglo XX (Sánchez et al. 2004). En efecto, Moreno et al. (2006) sugieren que en áreas con jaguares, las presas de tamaño pequeño, son más frecuentes en la dieta de los tigrillos que en aquellos sitios donde los jaguares han desaparecido, por lo tanto, la ausencia de competencia por presas con grandes carnívoros pudo haber permitido que el tigrillo ampliara su rango de tamaño de presas en la Reserva.

\section{AGRADECIMIENTOS}

Los autores quieren expresar su agradecimiento a Alberto Cadena, por su ayuda en la identificación de los mamíferos en la dieta del tigrillo y a Angélica Guzmán, por su colaboración en la separación de muestras. Rimma Gluhih y Dinesh Rao hicieron comentarios constructivos que ayudaron a mejorar el manuscrito. F. Sánchez agradece a los habitantes de la Reserva y a los ayudantes, por su apoyo durante el trabajo de campo y a la Fundación Gabriel Arango Restrepo, por el apoyo financiero al proyecto "Mamíferos de la Reserva Río Blanco".

\section{BIBLIOGRAFÍA}

ABREU, K.C.; MORO-RIOS, R.F.; SILVA-PEREIRA, j.e.; MIRANDA, J. M.D.; JABLONSKI, E.f.; PASSOS, F.C. 2007 Feeding habits of ocelot (Leopardus pardalis) in Southern Brazil. Mammalian Biology (en prensa).

ALBERICO, M.; CADENA, A.; HERNÁNDEZ-CAMACHO, J.; MUÑOZ-SABA, Y. 2000. Mamíferos (Synapsida: Theria) de Colombia. Biota Colombiana 1:43-75.

ALVEAR, M. 2000. Flora y vegetación de la Reserva Torre Cuatro (Manizales, Colombia). Tesis de pregrado inédita. Departamento de Biología, Universidad Nacional de Colombia (Bogotá).

ANÓNIMO. 1987. Cartografía integrada del medio natural: Chinchiná - Manizales. Análisis Geográficos - Instituto Geográfico Agustín Codazzi 8.

ARANDA, J.M. 1981. Rastros de los mamíferos silvestres de México: manual de campo. Instituto Nacional de Investigaciones sobre Recursos Bióticos (Xalapa, México). 198p.

BIANCHI, R.C.; MENDES, S.L. 2007. Ocelot (Leopardus pardalis) predation on primates in Caratinga Biological Station, Southeast Brazil. Am. J. Primatology. 69:1173-1178.

BISBAL, F.J. 1986. Food habits of some Neotropical carnivores in Venezuela (Mammalia, Carnivora). Mammalia. 50:329-337.

BISBAL, F.J. 1989. Distribution and habitat association of the carnivores in Venezuela. En: Redford, K.H.; 
Eisenberg, J.F., eds. Advances in Neotropical Mammalogy. The Sandhill Crame Press, Inc. (Gainesville, Fl.). p.339-362.

CAVELIER, J. 1995. Reforestation with the native tree Alnus acuminata: effects on phytodiversity and species richness in an upper mountain rain forest area of Colombia. En: Hamilton, L.S., Juvik, J.O.; Scatena, F.N., eds. Tropical montane cloud forest. Springer-Verlag, (New York). p.125-137.

CARLETON, M.D.; MUSSER, G.G. 1989. Systematic studies of Oryzomyinae rodents (Muridae, Sigmodontinae): a synopsis of Microryzomys. Bull. Am. Museum of Natural History. 191:1-83.

CHINCHILLA, F.A. 1997. La dieta del jaguar (Panthera onca), el puma (Felis concolor) y el manigordo (Felis pardalis) en el Parque Nacional Corcovado, Costa Rica. Rev. Biología Tropical. 45:1223-1229.

DE VILLA MESA, A.; MARTÍNEZ-MEYER, E.; LÓPEZGONZÁLEZ, C. 2002. Ocelot (Leopardus pardalis) food habits in a tropical deciduous forest of Jalisco, Mexico. Am. Midland Naturalist. 148:146-154.

EISENBERG, J.F. 1989. Mammals of the Neotropics: the northern Neotropics. Vol. 1. The University of Chicago Press (Chicago). 449p.

EMMONS, L.H. 1987. Comparative feeding ecology of felids in a Neotropical rainforest. Behavior, Ecology and Sociobiology. 20:271-283.

EMMONS, L.H. 1988. A field study of ocelots (Felis pardalis) in Peru. Revue d'Ecologie (Terre Vie). 43:133-157.

EMMONS, L.H. 1997. Neotropical rainforest mammals, $2^{\circ}$ ed. The University of Chicago Press (Chicago). 307p.

GÓMEZ-LAVERDE, M. 1994. Los pequeños mamíferos no voladores del Parque Regional Natural Ucumarí. En: Rangel J.O., ed. Ucumarí: Un caso típico de la diversidad biótica andina. CARDER (Corporación Autónoma Regional de Risaralda) - Instituto de Ciencias Naturales, Universidad Nacional de Colombia (Pereira). p.377-396.
GÓMEZ-VALENCIA, B. 2004. Estructura de la comunidad de pequeños mamíferos en un gradiente altitudinal, Reserva Río Blanco, Manizales-Caldas, Colombia. Tesis de pregrado inédita, Departamento de Biología, Universidad Nacional de Colombia (Bogotá). 98p.

HERNÁNDEZ-CAMACHO, J.I.; SÁNCHEZ, H. 1992. Biomas terrestres de Colombia. En: Halffter, G.C.C., ed. Diversidad biológica de Iberoamérica I. Instituto de Ecología (Xalapa, México). 390p.

JORGENSON, J.P.; RODRÍGUEZ-MAHECHA, J.V.; BEDOYA-GAITÁN, M.; DURÁN-RAMÍREZ, C.; GONZÁLEZ-HERNÁNDEZ, A. 2006. Tigrillo canaguaro Leopardus pardalis. En: Rodríguez-Mahecha, J.V.; Alberico, M.; Trujillo, F.; Jorgenson, J., eds. Libro rojo de los mamíferos de Colombia. Serie Libros Rojos de Especies Amenazadas de Colombia. Conservación Internacional Colombia, Ministerio de Ambiente, Vivienda y Desarrollo Territorial (Bogotá, Colombia). p.338-343.

KATTAN, G.H.; ÁLVAREZ-LÓPEZ, H. 1996. Preservation and management of biodiversity in fragmented landscapes in the Colombian Andes. En: Schelhas, J.; Greenberg, R., eds. Forest patches in tropical landscapes. Island Press (Washington, D. C.). p.3-18.

KONECNY, M.J. 1989. Movement patterns and food habits of four sympatric carnivore species in Belize, Central America. En: Redford, K.H.; Eisenberg, J.F., eds. Advances in Neotropical Mammalogy. The Sandhill Crane Press, Inc. (Gainesville, Fl.). p.243-264.

LUDLOW, M.E.; SUNQUIST, M.E. 1987. Ecology and behavior of ocelots in Venezuela. National Geographic Res. 3:447-461.

MONDOLFI, E. 1986. Notes on the biology and status of the small wild cats in Venezuela. En: Miller, S.D.; Everett, D.D., eds. Cats of the World: biology, conservation, and management. National Wildlife Federation (Washington, D.C.). p.125-146.

MORENO, R.S.; KAYS, R.W.; SAMUDIO, R., JR. 2006. Competitive release in diets of ocelot (Leopardus pardalis) 
and puma (Puma concolor) after jaguar (Panthera onca) decline. J. Mammalogy. 87:808-816.

MURCIA, C. 1997. Evaluation of Andean alder as a catalist for the recovery of tropical cloud forest in Colombia. Forest Ecology and Management. 99:163-170.

MURRAY, J.L.; GARDNER, G.L. 1997. Leopardus pardalis. Mammalian Species. 548:1-10.

RODRÍGUEZ-MAHECHA, J.V., HERNÁNDEZ-CAMACHO, J. I.; DEFLER, T.R.; ALBERICO, M.; MAST, R.B.; MITTERMEIER, R.A.; CADENA, A. 1995. Mamíferos colombianos: sus nombres comunes e indígenas. Vol. 3. Conservation International (Bogotá). 56p.

SÁNCHEZ, F.; ALVEAR, M. 2003. Comentarios sobre el uso de hábitat, dieta y conocimiento popular de los mamíferos en un bosque andino de Caldas, Colombia. Boletín Científico, Museo de Historia Natural, Universidad de Caldas 7:121-144.

SÁNCHEZ, F.; SÁNCHEZ-PALOMINO, P.; CADENA, A. 2004. Inventario de mamíferos en un bosque de los Andes centrales de Colombia. Caldasia. 26:291-309.

SÁNCHEZ, F.; SÁNCHEZ-PALOMINO, P.; CADENA, A. 2008. Species richness and indices of abundance of medium-sized mammals in an Andean forest and reforestations with Andean alder: a preliminary analysis. Caldasia. 30:197-208.

SUNQUIST, M.E.; SUNQUIST, F.; DANEKE, D.E. 1989. Ecological separation in a Venezuelan llanos carnivore community. En: Redford, K.H.; Eisenberg, J.F., eds. Advances in Neotropical Mammalogy. The Sandhill Crane Pres, Inc. (Gainesville, Florida, USA). p.197-232.

VOSS, R.S. 1988. Systematics and ecology of Ichthyomyine rodents (Muroidea): patterns of morphological evolution in a small adaptive radiation. Bull. Am. Museum of Natural History. 188:259-493.

VOSS, R.S. 2003. A new species of Thomasomys (Rodentia: Muridae) from eastern Ecuador, with remarks on mammalian diversity and biogeography in the Cordillera Oriental. Am. Museum Novitates. 3421:1-47.

WHITE, T.G.; ALBERICO, M.S. 1992. Dinomys branickii. Mammalian Species. 410:1-5.

WOZENCRAFT, W.C. 1993. Order Carnivora. En: Wilson, D.E.; Reeder, D. M., eds. Mammal species of the world. Smithsonian Institute Press (Washington, D.C.). p.286-346.

Recibido: Febrero 28 de 2008

Aceptado: Septiembre 8 de 2008 